\title{
Analysis of a Multiresonant Forward Converter Based on Nonideal Coupling of the Transformer
}

\author{
Trevor A. Smith, Student Member, IEEE, and Sima Dimitrijev, Member, IEEE
}

\begin{abstract}
There are many transformer applications where tight coupling is difficult to achieve. Therefore, an analysis of a resonant converter with a nonideal coupling is required. In this paper, a forward resonant converter with a coupling ranging from zero to one was analyzed. It was found that the traditional quasi-resonant converter becomes a multiresonant converter when the coupling is less than one. This is because the finite switching time of the rectifying diode reduces the input inductance of the transformer to effectively give a converter that uses two different inductances during any one switching period. It was also found that, in general, the converter has seven topological states. The design equations are derived analytically to provide a fundamental understanding of the converter. The peak voltage and current stress of the power switch as a function of the coupling were investigated. It was found that as the coupling is reduced, the peak voltage reduces and the peak current increases. A 48-5-V 20-W forward converter with a coupling of 0.9 was designed and tested. A coupling of 0.9 was chosen as it gives a duty cycle of $\mathbf{5 0 \%}$, has zero voltage switching for all loads, and has a peak switch voltage of 3.3 times the input voltage. The experiment verified the analysis and the practicality of a reduced coupling transformer, and the measured efficiency of the converter was $80 \%$.
\end{abstract}

Index Terms-DC-DC power conversion, equations, resonant power conversion, transformers.

\section{INTRODUCTION}

A COMMON goal of transformer designers has been to maximize the coupling. A high coupling is generally achieved when the magnetic material has a large permeability, when the air gaps in the material are kept small, and the windings are in close proximity to the material. Although a coupling of close to one is physically possible, it is becoming a more difficult task as converter frequencies increase. This is mainly because high frequency power magnetics have a lower permeability and the relative number of turns is decreasing. Therefore, a more complete analysis of a converter that includes the effects of coupling is required.

For the traditional analysis of resonant [1] and multiresonant [2]-[4] forward converters it is often convenient to model the transformer magnetizing inductance as a current source. This is only accurate if the magnetizing inductance is much larger than the leakage inductance, that is, if the transformer coupling is close to unity. Another common analysis assumption is that the

Manuscript received July 17, 1998; revised May 5, 1999. This work was supported by the Australian Research Council. Recommended by Associate Editor, K. D. T. Ngo.

T. A. Smith is with CSIRO Telecommunications and Industrial Physics, Lindfield, NSW, 2070, Australia (e-mail: trevor.smith@tip.csiro).

$\mathrm{S}$. Dimitrijev is with the School of Microelectronic Engineering, Griffith University, Nathan, Brisbane, QLD, 4111, Australia.

Publisher Item Identifier S 0885-8993(00)00377-X. rectifying and freewheeling diodes are ideal and their turn-on and turn-off times are instantaneous. This can be valid from a semiconductor point of view, but it is only accurate if the secondary leakage inductance is relatively small. This is because the rate of change of the rectifying diode current is governed by the secondary leakage inductance. Therefore, both of these assumptions cannot be used if the effects of transformer coupling are to be investigated.

Quasi-resonant converters use one inductor and one capacitor to achieve one resonant frequency [1]. The common multiresonant converter [2]-[4] uses one inductor and two capacitors to give more than one resonant frequency. This paper introduces the concept that a quasi-resonant forward converter actually becomes a multiresonant forward converter when the transformer coupling is less than one. This new converter uses one capacitor and effectively two inductors. The main advantage of this is that the nonideal coupling of a transformer can be exploited to give a very simple converter with a minimum parts count. The transformer can be less expensive and easier to construct, and the voltage stress on the switch can be reduced when compared to a quasi-resonant converter that has a wide load range. A typical quasi-resonant converter has a voltage stress that is proportional to the load range. For example, a fixed load can have a peak voltage as low as two times the input voltage, but a load range of $7: 1$ gives a peak voltage of eight times the input voltage [1]. A lower peak resonant voltage permits the use of a MOSFET with a lower on resistance or input capacitance, which is important for higher efficiency and simplified drive circuitry, respectively. This paper presents a multiresonant forward converter with a nonideal coupling and an experimental 48-5-V 20-W converter is designed and tested to confirm its practicality.

\section{THEORY OF OPERATION}

Fig. 1 shows a typical quasi-resonant forward converter where the transformer consists of two coupled inductors, the primary inductance $\left(L_{P}\right)$ and the secondary inductance $\left(L_{S}\right)$. Fig. 2(a) shows this transformer as a four terminal device. Fig. 2(b) shows an equivalent model of Fig. 2(a), derived from [5], which includes the transformer coupling $(k)$. Fig. 2(c) shows the equivalent primary input impedance $\left(Z_{I N P}\right)$ when the rate of change of current flowing out of terminal $c$ is constant. This occurs when the rectifying diode $\left(D_{R}\right)$ is fully on with the constant load current, or fully off with zero current. Fig. 2(d) shows $Z_{I N P}$ when there is a short circuit between terminals $\boldsymbol{c}$ and $\boldsymbol{d}$. This occurs when both $D_{R}$ and the freewheeling diode $\left(D_{F}\right)$ are conducting, such that their sum equals the load current. In this case, the voltage between 


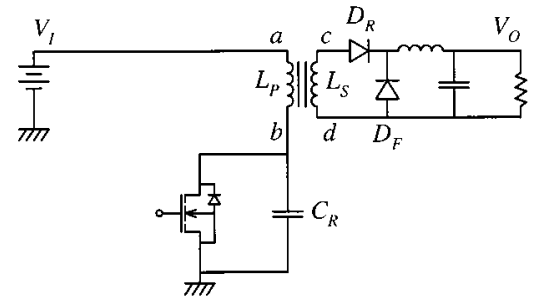

Fig. 1. A quasi-resonant forward converter.

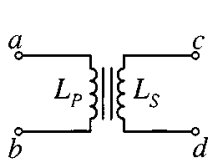

a)

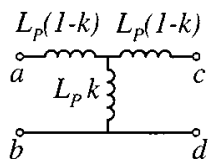

b)

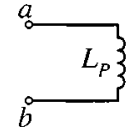

c)

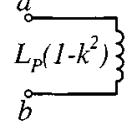

d)
Fig. 2. A transformer as (a) two coupled inductors and (b) an equivalent model. The transformer input inductance when (c) $D_{R}$ current is constant or zero and (d) $D_{R}$ current is changing.

terminals $\boldsymbol{c}$ and $\boldsymbol{d}$ is clamped at $0 \mathrm{~V}$ by the diodes to give a virtual short circuit.

Since $D_{R}$ can only be fully on, fully off or partly on, then Fig. 2(c) and (d) describes the two possible equivalent input impedances of the transformer. Therefore, there will be two different values of inductance resonating with the effective resonant capacitance $\left(C_{R}\right)$ during any one switching period. This gives rise to a multiresonant converter with two resonant frequencies of $1 / \sqrt{L_{P} C_{R}}$ and $1 / \sqrt{L_{P}\left(1-k^{2}\right) C_{R}}$, referred to as $\omega_{a}$ and $\omega_{b}$, respectively. $C_{R}$ can consist of an external capacitance in parallel with the output capacitance of the switch.

In general, there are seven states of operation for the converter. Fig. 3(a) shows the first five states for the voltage across the capacitor $\left(V_{C R}\right)$, the current through the primary inductance and the current through the rectifying diode. Fig. 3(b) and (c) shows the options for states six and seven. Fig. 4 is a flowchart of the seven states. It indicates the condition to enter a new state and whether the state is nonresonant or resonant at $\omega_{a}$ or $\omega_{b}$.

A steady-state description of the converter, with reference to Figs. 3 and 4, is as follows. In state 1 the switch is on, $D_{R}$ is fully on, $Z_{I N P}$ equals $L_{P}$ and $L_{P}$ has a linearly increasing current $\left(I_{L P}\right)$. The switch then turns off and the first resonant state, state 2 , begins. $D_{R}$ is still fully on so the resonant frequency is $1 / \sqrt{L_{P} C_{R}}$. Another resonant state, state 3 , begins when $D_{R}$ starts to turn off. This occurs when the voltage across the switch $\left(V_{C R}\right)$ equals the input voltage $\left(V_{I}\right)$. Here, the resonant frequency is $1 / \sqrt{L_{P}\left(1-k^{2}\right) C_{R}}$. The smaller equivalent inductance during this state lowers the peak resonant voltage when compared to a quasi-resonant converter. The next resonant state, state 4 , begins when $D_{R}$ is fully off, $D_{F}$ is now fully on and supplying the load current $\left(I_{O}\right)$. Since $D_{R}$ is fully off the resonant frequency here is $1 / \sqrt{L_{P} C_{R}}$. The peak resonant voltage occurs during this state. When $V_{C R}$ lowers from its peak back down to $V_{I}, D_{R}$ starts to conduct and a new resonant state, state 5, begins. Since $D_{R}$ and $D_{F}$ are both partly on, the resonant frequency is $1 / \sqrt{L_{P}\left(1-k^{2}\right) C_{R}}$. This state continues until $D_{R}$

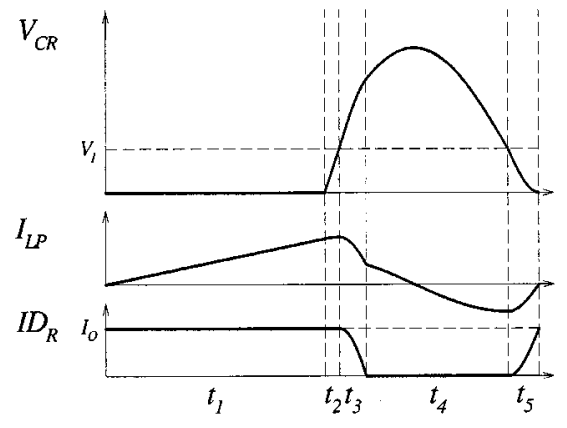

b)

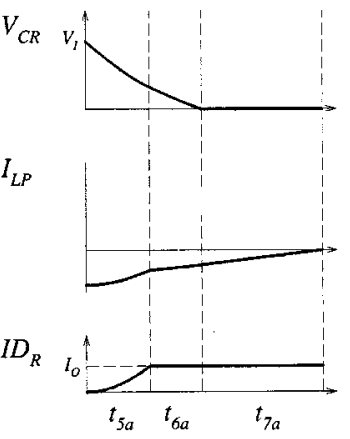

c)

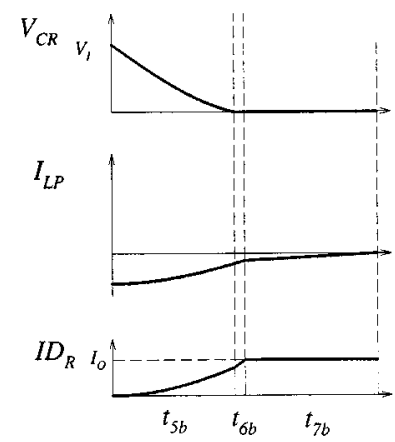

Fig. 3. $V_{C R}, I_{L P}$, and $I D_{R}$ for (a) the first five states (maximum power in this case), (b) states 5, 6, 7 for option $a$, and (c) states 5, 6, 7 for option $b$.

is fully on or $V_{C R}$ reaches $0 \mathrm{~V}$. If $D_{R}$ becomes fully on before $V_{C R}$ reaches $0 \mathrm{~V}$ then the new state, state $6 \mathrm{a}$, is resonant with $1 / \sqrt{L_{P} C_{R}}$, otherwise the state, state $6 \mathrm{~b}$, is nonresonant. State $6 \mathrm{a}$ and $6 \mathrm{~b}$ end when $V_{C R}$ reaches $0 \mathrm{~V}$ or $D_{R}$ reaches the load current $\left(I_{O}\right)$, respectively. The final state, state 7 , is when $I_{L P}$ decreases linearly back to zero. Here, $I_{L P}$ equals $V_{C R}$ which equals zero, and state one begins again. If in state $5, V_{C R}, I_{L P}$ and the current through $D_{R},\left(I D_{R}\right)$, simultaneously reach $0 \mathrm{~V}$, $0 \mathrm{~A}$, and $I_{O} \mathrm{~A}$, respectively, then states 6 and 7 do not exist. The output voltage is regulated for lighter loads by decreasing the on time of the switch. 


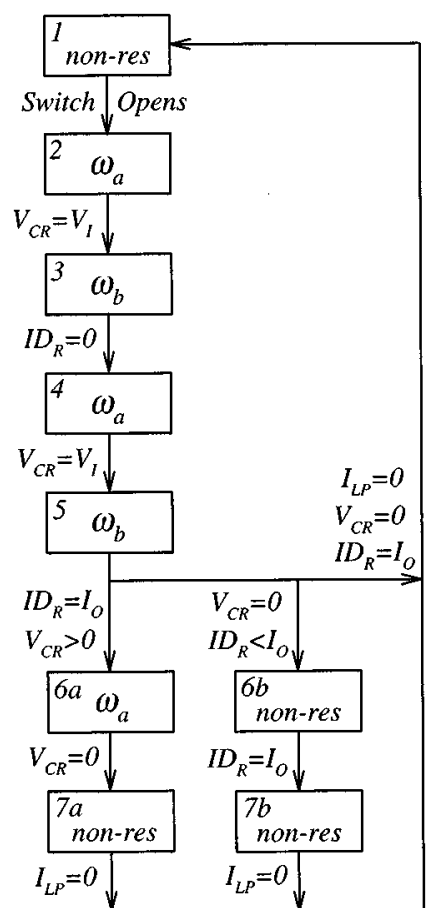

Fig. 4. Flowchart showing the sequence of states.

The turn-on and turn-off times of $D_{R}$ each introduce a new state. This transforms the quasi-resonant converter into a multiresonant converter. As $k$ becomes smaller, these two new states become more pronounced by occupying a larger percentage of the off time.

\section{ANALYSIS}

In general, the converter has seven states of operation. However, in this particular design, maximum power for the converter will require five states only, i.e., no states 6 or 7 , and can be seen in Fig. 3(a). States 6 and 7 will then exist when the output power is decreased from its maximum, i.e., when the load is reduced. Designing for maximum power using either six or seven states is also possible and will be studied in a future paper.

To simplify the analysis in the Appendix, a 100\% efficient converter was assumed. Some of the more common sources of power loss that were not included in the ideal analysis are copper ac/dc resistances, magnetic hysteresis and eddy currents, diode voltage drop, $R_{O N}$, and capacitor dielectrics.

The following equations for $L_{P}, L_{S}$, and $C_{R}$ are derived in the Appendix and $I_{O}$ refers to the maximum output current:

$$
\begin{gathered}
L_{P}=\frac{V_{I}^{2} t_{1}}{V_{O} I_{O}} f_{1}(k) \\
L_{S}=\frac{V_{O} t_{1}}{I_{O}} f_{2}(k) \\
C_{R}=\frac{V_{O} I_{O} t_{1}}{V_{I}^{2}} f_{3}(k)
\end{gathered}
$$

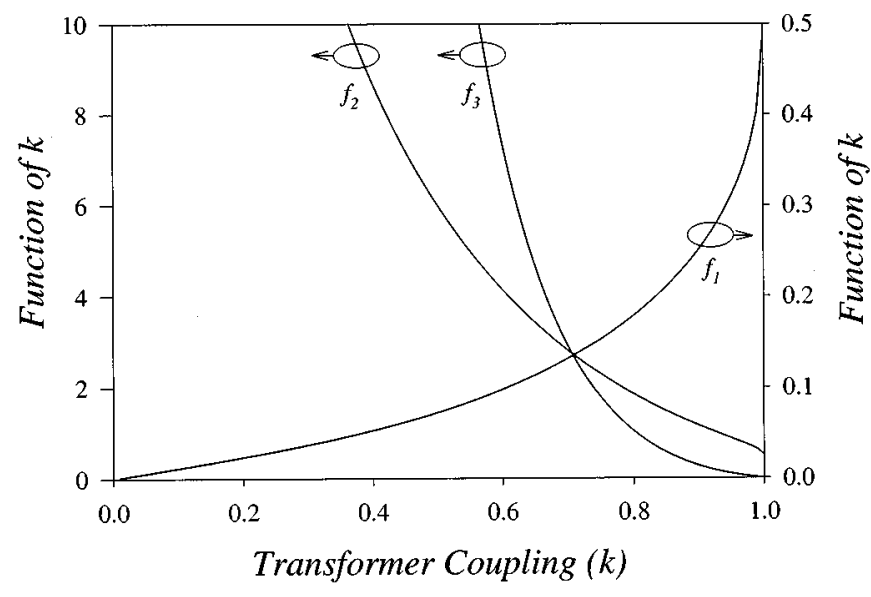

Fig. 5. Plots of $f_{1}, f_{2}$, and $f_{3}$ as universal functions of $k$.

where $f_{1}, f_{2}$, and $f_{3}$ are universal functions of $k$ only, and are plotted in Fig. 5.

Equations for maximum output power $\left(P_{O}\right)$ can be derived from these equations, from (1) and (3)

$$
P_{O}=\frac{V_{I}^{2}}{Z_{a}} f_{4}(k)
$$

where $Z_{a}$ is the characteristic impedance $Z_{a}=\sqrt{L_{P} / C_{R}}$, $f_{4}=\sqrt{f_{1} / f_{3}}$, and $P_{O}=V_{O} I_{O}$.

Since the analysis assumed a $100 \%$ efficient converter then $P_{O}$ must equal the input power $\left(P_{I}\right) . Z_{I}$ is the input impedance of the converter, from (4)

$$
P_{I}=\frac{V_{I}^{2}}{Z_{I}}
$$

where

$$
Z_{I}=\frac{Z_{a}}{f_{4}(k)}
$$

Therefore, the input impedance of the converter is simply the characteristic impedance multiplied by some function of $k$. It is interesting to note that $f_{4}(k)$ equals one when $k$ equals 0.9 . Since $f_{4}(k)$ is proportional to $k$ then $Z_{I}$ is inversely proportional to $k$. Therefore, $k$ effectively increases and decreases $Z_{I}$ when $k$ is less than or greater than 0.9 , respectively.

The voltage ratio $\left(V_{O} / V_{I}\right)$ can also be derived, from (1) and (2)

$$
\frac{V_{O}}{V_{I}}=\sqrt{\frac{L_{S}}{L_{P}}} f_{5}(k)
$$

where $f_{5}=\sqrt{f_{1} / f_{2}}$.

Therefore, the voltage ratio is simply the turns ratio multiplied by some function of $k$. Since $f_{5}(k)$ is proportional to $k$ then the voltage ratio is proportional to $k$. It is interesting to note that $f_{5}(k)$ equals one when $k$ equals one. This is not physically possible since a $k$ of one also implies a duty cycle of one. Therefore, the voltage ratio will always be less than the turns ratio, as in the original nonresonant forward converter [6]. In 


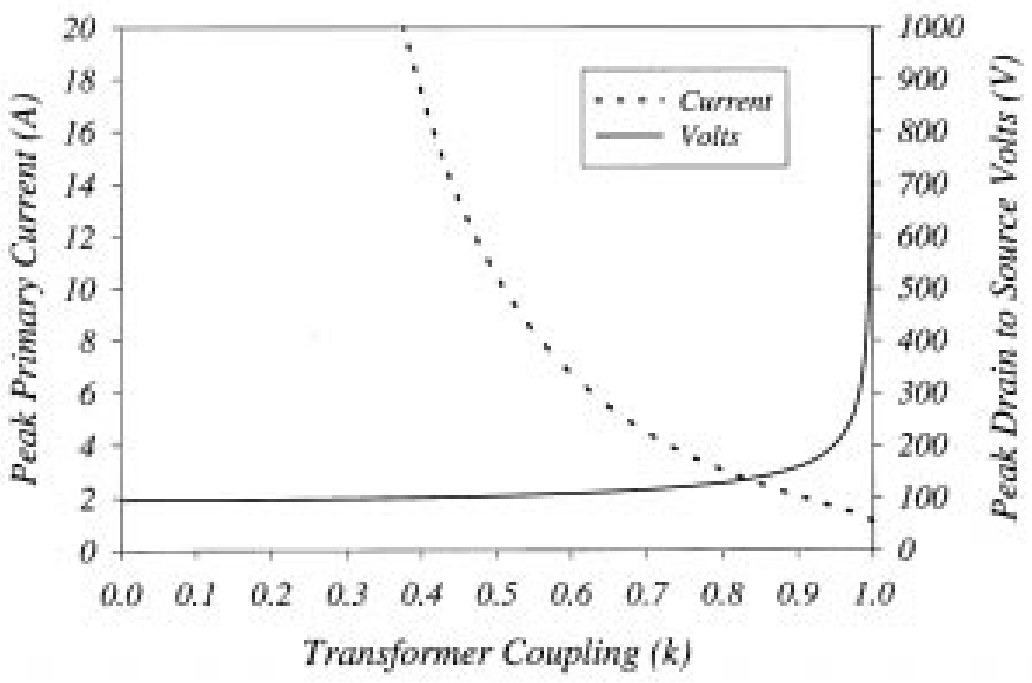

Fig. 6. The peak switch current and voltage as a function of $k$.

this case, the voltage ratio equals the turns ratio multiplied by the duty cycle as in (7)

$$
\frac{V_{O}}{V_{I}}=D \sqrt{\frac{L_{S}}{L_{P}}} .
$$

It is interesting to do a comparison of the new multiresonant forward converter with the original nonresonant one. It is stated in the Appendix that the time for each state can be expressed as functions of the on time and $k$. Therefore, the duty cycle $(D)$, which is a function of the state times, can be expressed purely as a function of $k$. When this is done it is found that it equals exactly twice $f_{1}(k)$. From (6)

$$
\frac{V_{O}}{V_{I}}=D \sqrt{\frac{L_{S}}{L_{P}}} f_{6}(k)
$$

where

$$
f_{6}=\frac{1}{\sqrt{4 f_{1} f_{2}}} .
$$

Therefore, the new multiresonant voltage ratio is the same as the original one, but multiplied by some function of $k . f_{6}(k)$ equals one when $k$ equals one and decreases to 0.707 when $k$ equals zero. Therefore, the new voltage ratio will always be less than the original one.

It appears then that the multiresonant converter presented in this paper has the same equations as the traditional nonresonant converters except that they are all multiplied by universal functions of $k$.

\section{EXPERIMENTAL INVESTIGATION}

To investigate the effects of $k$, the circuit of Fig. 1 was designed for a 48-5-V 20-W converter with an on time of 0.5 $\mu s$. The theoretical peak switch voltage and peak switch current were found as a function of $k$ and are shown in Fig. 6. It can be seen that a tradeoff between voltage stress and current stress exists, minimum current means maximum voltage, and vice versa. An optimum value of $k$ would depend solely on the application of the converter, and the relative effects for the whole system must be addressed. For example, lowering $k$ can lower the voltage stress on a MOSFET which in turn permits the use of a MOSFET with a lower on resistance $\left(R_{O N}\right)$. This would reduce the $I^{2} R$ loss. However, according to Fig. 6, the peak current stress is increased. This would increase the $I^{2} R$ loss. Other tradeoffs are also present, if $k$ is reduced then the required value of $L_{P}$ is reduced and that of $L_{S}$ and $C_{R}$ are increased. Therefore, if the reduction in parasitic resistance of $L_{P}$ is more substantial than the increase from $L_{S}$ and $C_{R}$ then the total $I^{2} R$ loss would be lower.

From Fig. 6, it can be seen that if $k$ is chosen to be approximately at the knee of the peak voltage curve, then at the expense of a slight increase in peak current, the peak voltage can be dramatically reduced. A $k$ of 0.9 was chosen to practically demonstrate the new multiresonant technique because it is near the knee and also corresponds to a duty cycle of $50 \%$. The duty cycle equals $50 \%$ when $f_{1}$ equals 0.25 , from Fig. 5 this occurs when $k$ is 0.9 .

Equations (1)-(3) were used to design a 48-5-V 20-W converter. $L_{P}, L_{S}$, and $C_{R}$ were found to be $14.4 \mu \mathrm{H}, 0.7 \mu \mathrm{H}$, and $1.2 \mathrm{nF}$, respectively. The analytical expressions for the waveforms from the Appendix were programmed in MATLAB and compared to a lossless circuit in PSPICE and an accurate match was found. The circuit was then built and all waveforms matched the theory accurately except that $V_{O}$ was only $4.2 \mathrm{~V}$. The on time could be increased from $0.5 \mu$ s to increase $V_{O}$ to $5 \mathrm{~V}$, but this increased the duty cycle and peak waveforms by approximately $50 \%$. It was then found that the main reason for $V_{O}$ being $4.2 \mathrm{~V}$ instead of $5 \mathrm{~V}$ was because of the diode voltage drop. This voltage drop needed to be included in the analysis to improve the accuracy and hence the practicality of the design equations. A method to include the voltage drop in the analysis without compromising its simplicity, is to add the forward voltage, in this case approximately $1 \mathrm{~V}$, to that required at the output while retaining the original output current. Therefore, a $48-5-\mathrm{V} 20-\mathrm{W}$ requirement means that a $48-6-\mathrm{V} 24-\mathrm{W}$ design should be implemented. This method increases the peak current 


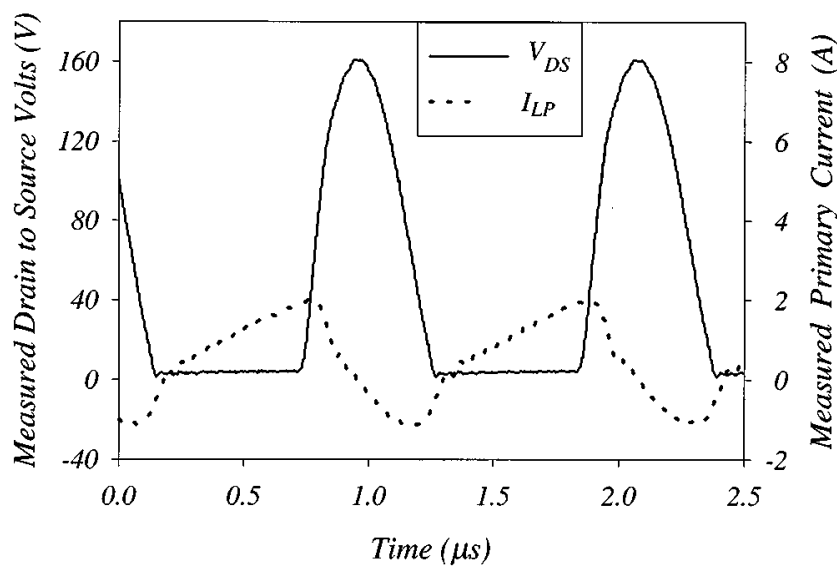

Fig. 7. Measured drain-to-source voltage and the primary current for an output of $20 \mathrm{~W}$.

slightly and the peak voltage remains the same. Fig. 6 is actually generated from a 48-6-V 24-W design.

Using (1)-(3) for a 48-6-V 24-W converter the circuit of Fig. 1 was redesigned and built using an IRF620 as the MOSFET. $C_{R}$ was a $1.3-\mathrm{nF}$ capacitor in parallel with the IRF620. $L_{P}$ was $12.0 \mu \mathrm{H}, L_{S}$ was $0.85 \mu \mathrm{H}$, and $k$ was 0.9 . The primary and secondary windings on a toroid were separated until a coupling of 0.9 was achieved. Lower coupling transformers can also be built by adding tuning inductors in series with the primary and secondary.

Fig. 7 shows the measured drain-to-source voltage $\left(V_{D S}\right)$, which is the same as $V_{C R}$, and the primary current $\left(I_{L P}\right)$. The practical and theoretical comparison of peak voltage, peak current and duty cycle matched exactly at $158 \mathrm{~V}, 2 \mathrm{~A}$, and 50\%, respectively. However, the on time was approximately $10 \%$ greater than predicted, and therefore, the switching frequency was $10 \%$ lower then predicted. It should be noted that the rise and fall times of the MOSFET gate voltage were each approximately $50 \mathrm{~ns}$ which leads to inaccuracies between the theoretical and practical on time. The on time can be increased or decreased slightly to compensate for inaccuracies and parasitics in the circuit. The efficiency was measured at $80 \%$.

Many converters require the output voltage to be regulated from full load to zero load. Regulation for lighter loads is achieved by decreasing the on time. ZVS might not always be achieved because reducing the on time also reduces the current, and hence energy, in the primary and secondary inductances prior to switching. A simple program utilizing the equations from the Appendix was implemented to investigate whether ZVS and output voltage regulation can be obtained for lighter loads. This was done by reducing the on time for each load until ZVS was just achieved. This gives the minimum output voltage where ZVS is still achieved. Fig. 8 shows this minimum voltage for couplings of $0.9,0.8,0.7$, and 0.6 . The figure shows that ZVS, or near ZVS, can be achieved for all loads when coupling is 0.9. Also observed from the figure is that ZVS, or near ZVS, for lighter loads becomes impossible as coupling decreases below 0.8 . This does not necessarily mean that the output cannot be regulated, but the efficiency would decrease. It should be noted that the theoretical curves near zero load

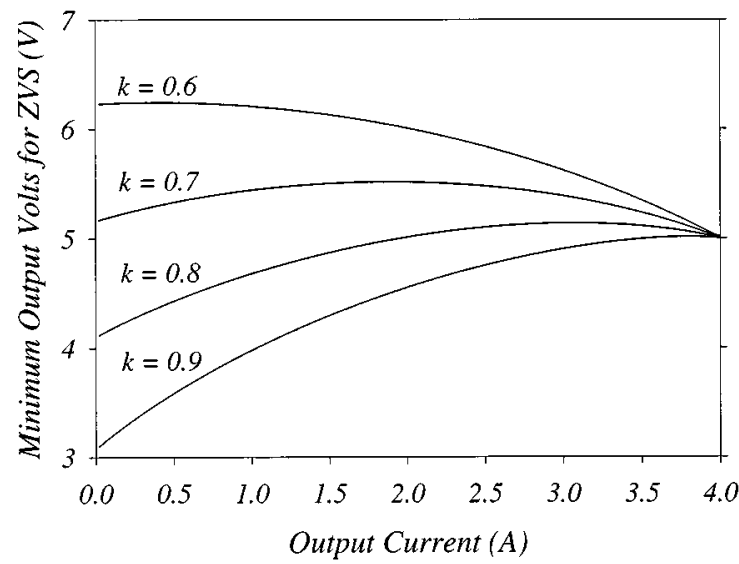

Fig. 8. Minimum output voltage that achieves ZVS for lighter loads.

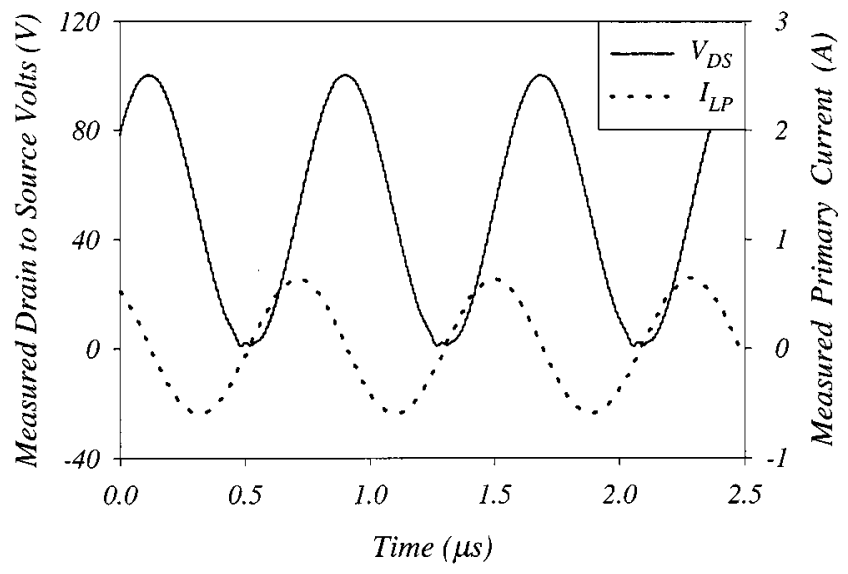

Fig. 9. Measured drain-to-source voltage and the primary current for an output of $2.5 \mathrm{~mW}$.

may not be achievable because these require an on time which is practically too small.

ZVS and an output voltage of $5 \mathrm{~V}$ was obtainable for all loads. Fig. 9 shows the measured $V_{D S}$ and $I_{L P}$ for an output power as low as $2.5 \mathrm{~mW}$.

\section{CONCLUSION}

Converter frequencies are increasing to facilitate size reduction and improved efficiency. As a result, obtaining near ideal transformer coupling is becoming increasingly difficult. A power supply that benefits from leakage inductances is required. In analyzing a forward converter with nonideal coupling, it was recognized that the magnetizing inductance should not be modeled with a current source, nor should it be assumed that the rectifying diode is switched instantaneously. When coupling is included in the analysis it is found that a quasi-resonant converter becomes a multiresonant converter which generally has seven states. The converter becomes multiresonant because there are effectively two inductors and one capacitor in any one switching period as opposed to the more common one inductor and two capacitors. The benefits of this are that the nonidealities of the transformer can be exploited and the peak voltage stress on the power switch can be reduced 
at the expense of a slight increase in the peak current. An experimental $48-5-\mathrm{V} 20-\mathrm{W}$ forward converter was designed and tested. The converter verified that by reducing the coupling of the transformer to 0.9 a practical and efficient converter with a realistic peak voltage and current can be designed.

\section{APPENDIX}

This Appendix derives the first order design equations for the converter. The analysis assumes there are no resistances in the circuit, i.e., $100 \%$ efficient, and that the output filter is ideal and there are no diode voltage drops. The design inputs and outputs are as follows.

1) Design Inputs:

$V_{I} \quad$ Input voltage.

$V_{O} \quad$ Output voltage.

$I_{O} \quad$ Output current.

$k$ Transformer coupling.

$t_{1} \quad$ On time of the switch.

2) Design Outputs-The Subscript $n$ Refers to the Relevant State:

$L_{P} \quad$ Value of the primary inductance.

$L_{S} \quad$ Value of the secondary inductance.

$C_{R} \quad$ Value of the resonant capacitor.

$I_{L n 0} \quad$ Initial current of $L_{P}$ for each state.

$V_{C n 0} \quad$ Initial voltage on $C_{R}$ for each state.

$I_{L n} \quad$ Current through $L_{P}$ for each state.

$V_{C n} \quad$ Voltage across $C_{R}$ for each state.

$t_{n} \quad$ Time for each state.

Each state of the circuit of Fig. 1 can be viewed on the primary side as an equivalent series LC circuit, this can be seen in Fig. 10. The relevant values for $L_{n}$ and $C_{n}$ will depend on the state, as per Figs. 3 and 4. They are given in Table I along with the known initial conditions. A value of infinity for $C_{n}$ indicates that the switch is on and the state is nonresonant. A value of $L_{P}\left(1-k^{2}\right)$ for $L_{n}$ indicates that the current through $D_{R}$ is changing as previously described in the theory of operation Fig. 2.

The general equations of Fig. 10 are [6]

$$
\begin{gathered}
I_{L n}=\left(\frac{V_{I}-V_{C n O}}{Z_{n}}\right) \sin \left(\omega_{n} t\right)+I_{L n 0} \cos \left(\omega_{n} t\right) \\
V_{C n}=V_{I}+\left(V_{C n O}-V_{I}\right) \cos \left(\omega_{n} t\right)+I_{L n 0} Z_{n} \sin \left(\omega_{n} t\right)
\end{gathered}
$$

where

$$
\begin{gathered}
Z_{n}=\sqrt{\frac{L_{n}}{C_{n}}} \\
\omega_{n}=\frac{1}{\sqrt{L_{n} C_{n}}} .
\end{gathered}
$$

Since $Z_{n}$ and $\omega_{n}$ each have only two possible values, then $Z_{a}$, $Z_{b}, \omega_{a}$, and $\omega_{b}$ will be defined as

$$
Z_{a}=\sqrt{\frac{L_{P}}{C_{R}}}
$$

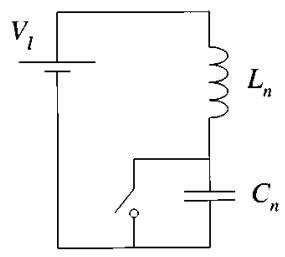

Fig. 10. A series LC circuit with a switch.

TABLE I

INDUCTANCE AND CAPacitance VAlues WITH THEIR KNOWN INITIAL CONDITIONS FOR EACH STATE

\begin{tabular}{c|c|c|c|c}
\hline State $(\boldsymbol{n})$ & $\boldsymbol{L}_{\boldsymbol{n}}$ & $\boldsymbol{I}_{L \boldsymbol{n} 0}$ & $\boldsymbol{C}_{\boldsymbol{n}}$ & $V_{C n 0}$ \\
\hline 1 & $L_{P}$ & 0 & $\infty$ & 0 \\
\hline 2 & $L_{P}$ & $I_{L 20}$ & $C_{R}$ & 0 \\
\hline 3 & $L_{P}\left(I-k^{2}\right)$ & $I_{L 30}$ & $C_{R}$ & $V_{I}$ \\
\hline 4 & $L_{P}$ & $I_{L 40}$ & $C_{R}$ & $V_{C 40}$ \\
\hline 5 & $L_{P}\left(I-k^{2}\right)$ & $I_{L 50}$ & $C_{R}$ & $V_{I}$ \\
\hline $6 \mathrm{a}$ & $L_{P}$ & $I_{L 6 a 0}$ & $C_{R}$ & $V_{C 6 a 0}$ \\
\hline $6 \mathrm{~b}$ & $L_{P}\left(I-k^{2}\right)$ & $I_{L b b 0}$ & $\infty$ & 0 \\
\hline $7 \mathrm{a}$ & $L_{P}$ & $I_{L 7 a 0}$ & $\infty$ & 0 \\
\hline $7 \mathrm{~b}$ & $L_{P}$ & $I_{L 7 b 0}$ & $\infty$ & 0 \\
\hline
\end{tabular}

$$
\begin{gathered}
Z_{b}=\sqrt{\frac{L_{P}\left(1-k^{2}\right)}{C_{R}}} \\
\omega_{a}=\frac{1}{\sqrt{L_{P} C_{R}}} \\
\omega_{b}=\frac{1}{\sqrt{L_{P}\left(1-k^{2}\right) C_{R}}} .
\end{gathered}
$$

3) Equations for Current and Voltage with Initial Conditions: The following rules will be used in the analysis and referred to where applicable:

$$
\begin{aligned}
\pm A \cos (\phi)+B \sin (\phi)= & \pm \sqrt{A^{2}+B^{2}} \cos \\
& \cdot\left(\phi-\tan ^{-1}\left(\frac{B}{A}\right)\right)
\end{aligned}
$$

$$
\cos \left(\sin ^{-1}(\alpha)\right)=\sin \left(\cos ^{-1}(\alpha)\right)=\sqrt{1-\alpha^{2}}
$$

$$
\tan ^{-1} \frac{A}{B}+\tan ^{-1} \frac{B}{A}=\frac{\pi}{2}
$$

$$
\sin \left(\tan ^{-1}(\alpha)\right)=\frac{\alpha}{\sqrt{1+\alpha^{2}}}
$$

$$
\cos \left(\tan ^{-1}(\alpha)\right)=\frac{1}{\sqrt{1+\alpha^{2}}} .
$$


The following equations for $I_{L n}$ and $V_{C n}$ are derived by substituting the relevant values from Table I into (A1) and (A2):

$$
\begin{gathered}
I_{L 1}=\frac{V_{I}}{L_{P}} t \\
V_{C 1}=0 \\
I_{L 2}=\frac{V_{I}}{Z_{a}} \sin \left(\omega_{a} t\right)+I_{L 20} \cos \left(\omega_{a} t\right) \\
V_{C 2}=V_{I}-V_{I} \cos \left(\omega_{a} t\right)+I_{L 20} Z_{a} \sin \left(\omega_{a} t\right) \\
V_{C 6 a}=V_{I}+\left(V_{C 6 a 0}-V_{I}\right) \cos \left(\omega_{a} t\right)+I_{L 6 a 0} Z_{a} \sin \left(\omega_{a} t\right) \\
I_{L 63}=I_{L 30} \cos \left(\omega_{b} t\right) \\
I_{L 4}=\left(\frac{V_{I}-V_{C 40}}{Z_{a}}\right) \sin \left(\omega_{a} t\right)+I_{L 40} \cos \left(\omega_{a} t\right) \\
V_{C 4}=V_{I}+\left(V_{C 40}-V_{I}\right) \cos \left(\omega_{a} t\right)+I_{L 40} Z_{a} \sin \left(\omega_{a} t\right) \\
\text { (A9) }
\end{gathered}
$$$$
I_{L 6 b}=\frac{V_{I}}{L_{P}\left(1-k^{2}\right)} t+I_{L 6 b 0}
$$$$
V_{C 6 b}=0
$$$$
I_{L 7 a}=\frac{V_{I}}{L_{P}} t+I_{L 7 a 0}
$$$$
V_{C 7 a}=0
$$

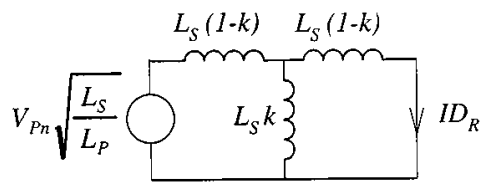

Fig. 11. Equivalent secondary side circuit during states 3 and 5.

$$
\begin{gathered}
I_{L 7 b}=\frac{V_{I}}{L_{P}} t+I_{L 7 b 0} \\
V_{C 7 b}=0 .
\end{gathered}
$$

4) Equations for Time with Initial Conditions: The time for a state $t_{n}$ is found by equating either $I_{L n}$ or $V_{C n}$ to $I_{L(n+1) 0}$ or $V_{C(n+1) 0}$, respectively, and then solving for $t$.

Time $1-t_{1}$ : Although $t_{1}$ is an input parameter, its relationship to $L_{P}$ is still required, from (A3)

$$
t_{1}=\frac{I_{L 20}}{V_{I}} L_{P}
$$

Time $2-t_{2}$ : From (A6) and (R1)

$$
t_{2}=\frac{1}{\omega_{a}} \tan ^{-1}\left(\frac{V_{I}}{I_{L 20} Z_{a}}\right) .
$$

Time $3-t_{3}: t_{3}$ is the time taken for $I D_{R 3}$ to go from $I_{O}$ to zero. To find $t_{3}$ an equation for $I D_{R 3}$ has to be found. $t_{3}$ is then found by equating $I D_{R 3}$ to zero. The equivalent transformer model, when referred to the secondary, can also be derived from [5] and it is the same as Fig. 2(b), but replacing $L_{P}$ with $L_{S}$. The voltage between terminals $\boldsymbol{a}$ and $\boldsymbol{b}\left(V_{a b}\right)$ will now be the primary voltage $\left(V_{P n}\right)$ multiplied by the turns ratio, subscript $n$ is the state number

$$
V_{a b}=V_{P_{n}} \sqrt{\frac{L_{S}}{L_{P}}} .
$$

Fig. 11 shows the equivalent circuit to solve for $I D_{R 3}$ and hence $t_{3}$. Since $D_{F}$ is starting to conduct at the beginning of $t_{3}$ then both diodes are on and the voltage between terminals $\boldsymbol{c}$ and $\boldsymbol{d}$ is clamped at zero, i.e., short circuit. At the beginning of $t_{3}, I D_{R}$ equals $I_{O}$ so the secondary leakage inductance, $L_{S}(1-k)$, has an initial condition of $I_{O}$. Since at the beginning of $t_{3}, V_{P}$ equals zero and $I_{L 30}$ is at a peak and constant, there will be no initial current through the reflected inductance, $L_{S}(1-k)$. Therefore, the secondary magnetizing inductance must also have an initial condition of $I_{O}$. From (A8), Fig. 1, and Fig. 11

$$
I D_{\mathrm{R} 3}=\frac{-I_{L 30} Z_{b} k}{\omega_{b} L_{S}\left(1-k^{2}\right)} \sqrt{\frac{L_{S}}{L_{P}}}\left(1-\cos \left(\omega_{b} t\right)\right)+I_{O} .
$$

From (A24)

$$
t_{3}=\frac{1}{\omega_{b}}\left(\cos ^{-1}\left(1-\frac{I_{O}}{k I_{L 30}} \sqrt{\frac{L_{S}}{L_{P}}}\right)\right) .
$$


Time 4-t4: From (A10) and (R1)

$$
t_{4}=\frac{1}{\omega_{a}}\left(\frac{\pi}{2}+\tan ^{-1}\left(\frac{I_{L 40} Z_{a}}{V_{C 40}-V_{I}}\right)\right) .
$$

Time $5 a-t_{5 a}: t_{5 a}$ is the time taken for $I D_{R}$ to go from zero to $I_{O} . t_{5 a}$ is found the same way as $t_{3}$ except that the initial currents in the secondary leakage and magnetizing inductances are zero and $V_{P n}$ equals $V_{P 5} . t_{5}$ is then found by setting $I D_{R}$ equal to $I_{O}$. This gives

$$
t_{5 a}=\frac{1}{\omega_{b}}\left(\cos ^{-1}\left(1+\frac{I_{O}}{k I_{L 50}} \sqrt{\frac{L_{S}}{L_{P}}}\right)\right) .
$$

Time $5 b-t_{5 b}:$ From (A12)

$$
t_{5 b}=\frac{1}{\omega_{b}} \sin ^{-1}\left(\frac{-V_{I}}{I_{L \check{ }} Z_{b}}\right) .
$$

Time $6 a-t_{6 a}:$ From (A14) and (R1)

$$
\begin{aligned}
t_{6 a}=\frac{1}{\omega_{a}} & \left(\cos ^{-1}\left(\frac{-V_{I}}{\sqrt{\left(V_{C 6 a 0}-V_{I}\right)^{2}+\left(I_{L 6 a 0} Z_{a}\right)^{2}}}\right)\right. \\
& \left.-\frac{\pi}{2}-\tan ^{-1}\left(\frac{V_{C 6 a 0}-V_{I}}{I_{L 6 a 0} Z_{a}}\right)\right) .
\end{aligned}
$$

Time $6 b-t_{6 b}$ : State $6 \mathrm{~b}$ occurs because $V_{C 5}$ reaches zero before $I D_{R}$ reaches $I_{O} . t_{6 b}$ is the time taken for $I D_{R 6 b}$ to go from $I D_{R 6 b 0}$ to $I_{O}$. In state $6 \mathrm{~b}, V_{P 6 b}$ equals $V_{I}$ and the voltage across the primary magnetizing inductance $\left(V_{P M}\right)$ equals $\frac{k V_{I}}{k+1}$. Therefore, the voltage across the secondary magnetizing inductance $\left(V_{S M}\right)$ is, from (Fig. 11)

$$
V_{S M}=\frac{k V_{I}}{k+1} \sqrt{\frac{L_{S}}{L_{P}}}
$$

Since both diodes are on, then $V_{S M}$ also falls across the secondary leakage inductance, $L_{S}(1-k)$. $\therefore$ from (A30)

$$
I D_{R 6 B}=\frac{k V_{I}}{L_{S}\left(1-k^{2}\right)} \sqrt{\frac{L_{S}}{L_{P}}} t+I D_{R 6 b 0}
$$

where $I D_{R 6 b 0}$ equals $I D_{R \tilde{5}}$ at the end of $t_{\check{5} b}$.

From (A12), (A28), (R2), Fig. 1, and Fig. 11

$I D_{R 6 b 0}=\frac{-k I_{L 50} Z_{b}}{\omega_{b} L_{S}\left(1-k^{2}\right)} \sqrt{\frac{L_{S}}{L_{P}}}\left(1-\sqrt{1-\left(\frac{V_{I}}{I_{L 50} Z_{b}}\right)^{2}}\right)$.

From (A31) and (A32)

$$
\begin{aligned}
t_{6 b}= & \frac{I_{O} L_{S}\left(1-k^{2}\right)}{k V_{I}} \sqrt{\frac{L_{P}}{L_{S}}}+\frac{I L_{50} L_{P}\left(1-k^{2}\right)}{V_{I}} \\
& \cdot\left(1-\sqrt{1-\left(\frac{V_{I}}{I L_{50} Z_{b}}\right)^{2}}\right) .
\end{aligned}
$$

Time $7 a-t_{7 a}: t_{7}$ is the time taken for $I_{L 7}$ to reach zero. From (A17)

$$
t_{7 a}=\frac{-I_{L 7 a 0} L_{P}}{V_{I}}
$$

Time $7 b-t_{7 b}:$ From (A19)

$$
t_{7 b}=\frac{-I_{L 7 b 0} L_{P}}{V_{I}} .
$$

5) Initial Conditions: If the initial conditions $I_{L n 0}$ and $V_{C n 0}$ cannot be found from Figs. 3 and 4 then they are found by substituting $t_{n-1}$ into either $I_{L n-1}$ or $V_{C n-1}$. From Figs. 3 and 4

$$
\begin{aligned}
& I_{L 10}=0 \quad V_{C 10}=0 \quad V_{C 20}=0 \quad V_{C 30}=V_{I} \\
& V_{C 50}=V_{I} \quad V_{C 660}=0 \quad V_{C 7 a 0}=0 .
\end{aligned}
$$

From (A3)

$$
I_{L 20}=\frac{V_{I}}{L_{P}} t_{1} .
$$

From (A5), (A22), and (R1)

$$
I_{L 30}=\sqrt{I_{L 20}^{2}+\left(\frac{V_{I}}{Z_{a}}\right)^{2}} .
$$

From (A7) and (A25)

$$
I_{L 40}=I_{L 30}-\frac{I_{O}}{k} \sqrt{\frac{L_{S}}{L_{P}}} .
$$

From (A8), (A25), (A39), and (R2)

$$
V_{C 40}=V_{I}+Z_{b} \sqrt{I_{L 30}^{2}-I_{L 40}^{2}} .
$$

From (A9), (A25), (A26), (R1), and (R3)

$$
I_{L 50}=-\sqrt{I_{L 40}^{2}+\left(\frac{V_{I}-V_{C 40}}{Z_{a}}\right)^{2}} .
$$

From (A11) and (A27)

$$
I_{L 6 a 0}=I_{L \tilde{0} 0}+\frac{I_{O}}{k} \sqrt{\frac{L_{S}}{L_{P}}} .
$$

From (A27) and (R2)

$$
V_{C 6 a 0}=V_{I}-Z_{b} \sqrt{I_{L 50}^{2}-I_{L 6 a 0}^{2}}
$$

From (A11), (A12), (A28), and (R2)

$$
I_{L 6 b 0}=-\sqrt{I_{L 50}^{2}-\left(\frac{V_{I}}{Z_{b}}\right)^{2}} .
$$

From (A29) and (R2)

$$
I_{L 7 a 0}=-\sqrt{I_{L 6 a 0}^{2}+V_{C 6 a 0}^{2}-2 V_{I} V_{C 6 a 0}} .
$$

From (A13), (A33), and (A15)

$$
I_{L 7 b 0}=\frac{I_{O}}{k(1+k)} \sqrt{\frac{L_{S}}{L_{P}}}+\frac{I_{L \check{0} 0}-I_{L 6 b 0}}{(1+k)}+I_{L 6 b 0} .
$$

6) Calculating $L_{P}, L_{S}$, and $C_{R}$ : In this section, expressions for $L_{P}, L_{S}$, and $C_{R}$ will be calculated such that maximum power is delivered in five states as seen in Fig. 3(a). It will be seen that there are three unique equations for $I_{L 50}$ and one for 
$V_{O}$. These four equations will contain four unknowns $L_{P}, L_{S}$, $C_{R}$, and $I_{L 50}$.

The design uses zero voltage switching and zero current switching at the maximum output power as a boundary condition. Therefore, when $V_{C \Sigma}$ equals zero then $I_{L \tilde{L}}$ equals zero and $I D_{R}$ equals $I_{O}$.

7) Equations for $I_{L 50}$ : To make $V_{C 5}$ equal zero when $I_{L 5}$ equals zero means that the minimum of $V_{C 5}$ equals zero. This means that the peak ac voltage plus the dc voltage has to equal zero. It should be noted that if the output power is increased beyond the design limit then ZVS will no longer be possible. From (A12)

$$
I_{L 50}=\frac{-V_{I}}{Z_{b}}
$$

The boundary condition for maximum power is that the peak ac voltage equals the dc voltage. Therefore, the time for state 5 is actually one quarter of the period of the appropriate resonant frequency. The resonant frequency of state 5 is $\omega_{b}$. That is,

$$
t_{5}=\frac{\pi}{2 \omega_{b}} .
$$

$I D_{R}$ equals $I_{O}$ when $V_{C 5}$ equals zero is also a boundary condition at maximum power. Therefore, since $t_{5 a}$ is the time for $I D_{R}$ to go from zero to $I_{O}$ then $t_{5 a}$ must also be one quarter of the period. From (A27) and (A48)

$$
I_{L 50}=\frac{-I_{O}}{k} \sqrt{\frac{L_{S}}{L_{P}}} .
$$

From (A37) to (A41), see (A50), given at the bottom of the page.

8) Equation for $V_{O}: V_{O}$ is the dc component, or average, of the voltage across $D_{F}\left(V D_{F}\right)$. When $I D_{R}$ is zero or changing, states 3-5, then $V D_{F}$ is zero. When $I D_{R}$ equals $I_{O}$ and is constant, states 1 and $2, V D_{F}$ does not equal zero. There is no voltage drop across the secondary leakage inductance when $I D_{R}$ is constant

$$
\begin{array}{cc}
V D_{F n}=V_{S M n} & (n=1,2) \\
V D_{F n}=0 & (n=3,4,5) \\
V_{S M n}=k V_{P M n} \sqrt{\frac{L_{S}}{L_{P}}} \\
V_{P M n}=V_{I}-V_{C n} .
\end{array}
$$

From (A51) to (A53)

$$
V D_{F n}=k\left(V_{I}-V_{C n}\right) \sqrt{\frac{L_{S}}{L_{P}}} \quad(n=1,2)
$$

$$
V D_{F n}=0 \quad(n=3,4,5) .
$$

The dc component is then

$$
V_{O}=\frac{\sum_{n=1 \text { to } 5} \int_{0}^{t_{n}} V D_{F n} d t}{\sum_{n=1 \text { to } 5} t_{n}} .
$$

From (A2a), (A2c), (A3), (A4), (A6), (A54), (A55), (R4), and (R5)

$$
V_{O}=\frac{k V_{I} \sqrt{L_{S} C_{R}+\frac{L_{S}}{L_{P}} t_{1}^{2}}}{t_{1}+t_{2}+t_{3}+t_{4}+t_{5}}
$$

where $t_{2}-t_{\tilde{5}}$ can be expressed as functions of $t_{1}$ and $k$.

9) Solution of the Four Equations: The three equations for $I_{L 50}$ (A47), (A49), and (A50) and the one equation for $V_{O}$ (A56) can be solved by substitution. This yields the equations for $L_{P}$, $L_{S}$, and $C_{R}$

$$
\begin{gathered}
L_{P}=\frac{V_{I}^{2} t_{1}}{V_{O} I_{O}} f_{1}(k) \\
L_{S}=\frac{V_{O} t_{1}}{I_{O}} f_{2}(k) \\
C_{R}=\frac{V_{O} I_{O} t_{1}}{V_{I}^{2}} f_{3}(k)
\end{gathered}
$$

where $f_{1}, f_{2}$, and $f_{3}$ are only functions of $k$, i.e., do not depend on any other parameters, and are plotted in Fig. 5 in the analysis section. Equations for input power, output power, and voltage ratio are derived in the analysis section.

\section{REFERENCES}

[1] W. A. Tabisz, P. M. Gradzki, and F. C. Lee, "Zero-voltage-switched quasiresonant buck and flyback converters-Experimental results at 10MHz," IEEE Trans. Power Electron., vol. 4, pp. 194-204, Apr. 1989.

[2] W. A. Tabisz and F. C. Lee, " $5 \mathrm{MHz}, 50 \mathrm{~W}$, zero-voltage switched multiresonant converter," in HFPC, 1988.

[3] W. Tang, W. A. Tabisz, A. Lofti, F. C. Lee, and V. Vorperian, "DC analysis and design of forward multi-resonant converter," in IEEE PESC, 1990, pp. 862-869.

[4] W. Tang, C. S. Leu, and F. Lee, "Charge control for zero-voltageswitching multiresonant converter," IEEE Trans. Power Electron., vol. 11, pp. 270-274, Mar. 1996.

[5] G. McPherson and R. Laramore, An Introduction to Electrical Machines and Transformers, 2nd ed. New York, NY: Wiley, 1990.

[6] N. Mohan, T. M. Underland, and W. P. Robbins, Power Electronics-Converters, Applications, and Design, 2nd ed. New York, NY: Wiley, 1995.

$$
I_{L \check{5} 0}=-\sqrt{\left(\frac{V_{I} t_{1}}{L_{P}}\right)^{2}+\left(\frac{V_{I}}{Z_{a}}\right)^{2}-2 k I_{O} \sqrt{\frac{L_{S}}{L_{P}}} \sqrt{\left(\left(\frac{V_{I} t_{1}}{L_{P}}\right)^{2}+\left(\frac{V_{I}}{Z_{a}}\right)^{2}\right)}+I_{O}^{2} \frac{L_{S}}{L_{P}}}
$$




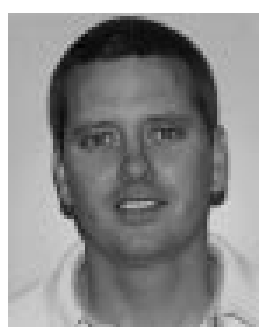

Trevor A. Smith (S'93) was born in Brisbane, Australia, in 1967. He received the Bachelor of Engineering degree in microelectronic engineering with a major in communication (first class honors) from Griffith University, Brisbane, in 1996. He is currently working toward the Ph.D. degree at Griffith University.

His interests include resonant converters, transformer design, and electromagnetics.

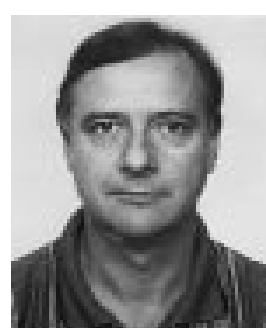

Sima Dimitrijev (S'87-M'88) received the B.Eng., M.Sci., and Ph.D. degrees in electronic engineering from the University of Nis, Yugoslavia, in 1982, 1985 , and 1989 , respectively.

From 1982 to 1983 , he was with the Semiconductor Factory of the Electronics Industry, Nis, where he worked on the development of CMOS technology. From 1983 to 1990 , he was with the Faculty of Electronic Engineering at the University of Nis. In 1990, he joined Griffith University, Brisbane, Australia, where he is currently an Associate Professor at the School of Microelectronic Engineering. He is an active researcher in the areas of semiconductor technology (including micromachining and integrated optics), MOSFET applications (in particular power electronics), as well as MOSFET design, modeling, fabrication, and characterization (including SiC MOSFET's). He is a member of the Editorial Board of Microelectronics Reliability and the author of Understanding Semiconductor Devices (New York: Oxford University Press). 